\title{
Drops that Change their Mind: Spontaneous Reversal from
}

\section{Spreading to Retraction}

\author{
R. Tadmor ${ }^{*}, 1,2$, A. Baksi ${ }^{1}$, S. Gulec ${ }^{1}$, S. Jadhav ${ }^{1}$, H.E. N'guessan ${ }^{1}$, K. Sen ${ }^{1}$, V. Somasi ${ }^{1}$, M. \\ Tadmor $^{1}$, P. Wasnik ${ }^{1}$, S. Yadav ${ }^{1}$ \\ * Corresponding Author. Email: rafael.tadmor@lamar.edu and tadmorr@bgu.ac.il
}

1. Dan F. Smith Department of Chemical Engineering, Lamar University, Beaumont, TX 77710.

2. Department of Mechanical Engineering, Ben Gurion University, Beer Sheva, Israel

The premise of our paper is the retraction that follows the spreading. Yet, here we also discuss the spreading part. The Young equation describes the contact angle a drop makes with a surface as follows:

$\cos \theta=\left(\gamma_{S V}-\gamma_{S L}\right) / \gamma_{L V}$

Where $\theta$ is the contact angle a drop adopts on a surface, $\gamma_{S V}, \gamma_{S L}$ and $\gamma_{L V}$ are the interfacial tension between solid-vapor, solid-liquid and liquid-vapor, respectively.

One can see that if $\left(\gamma_{S V}-\gamma_{S L}\right)>\gamma_{L V}$, the equation cannot be solved for $\theta$. In such cases, the liquid drop will spread as long as there is solid surface available for spreading, and the system is often defined by the Harkin spreading coefficient ${ }^{1}, \pi_{e}$, where $\pi_{e}=\gamma_{S V}-\gamma_{S L}-\gamma_{L V}$ describes the tendency of a liquid to spread on another liquid film or a solid surface. Since,

$$
\gamma_{\text {mica }}>>\gamma_{T D}+\gamma_{S A M / T D}
$$


Then the ODA/TD liquid spreads on a freshly cleaved mica surface and theoretically should continue to spread. Yet, we show that, after a short spreading it retracts. Apart from surface tensions, the spreading kinetics also depends on the viscosity of the liquid, and the literature usually considers it in terms of Tanner's law (following a first fast spreading when the drop first coalescence with the solid surface). In the Tanner regime, the radius of the spreading drop increases as a power law of the time,

$R \sim t^{n}$

where, $R$ is the radius of the spreading drop, $t$ is the time from the moment the drop was placed on the solid surface, and $n$ is a positive number. For many cases $n=1 / 10$. Since we have subsequent retraction, our spreading law is short lived. Nonetheless, it seems that our spreading does not follow a classic Tanner law. For example, Fig. S1 shows the spreading of a $4 \mu$ drop of $1.2 \mathrm{mM}$ ODA/TD up to the time when it is truncated by the retraction, along with the power law: $R=2 *$ time $^{0.1}$. We see that in general the curve does not follow the classic Tanner's law.

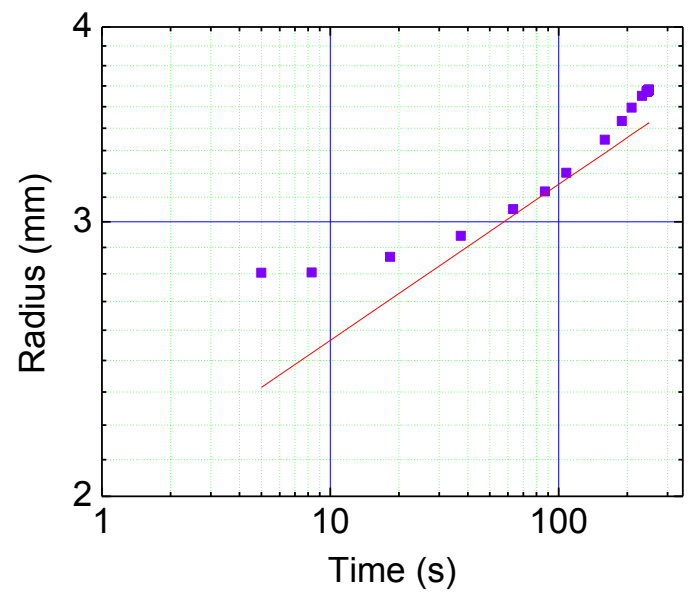


Figure S1: Solid-liquid radius versus time of a $4 \mu 1.2 \mathrm{mM}$ ODA/TD drop, during its spreading on a freshly cleaved mica surface.

\section{Values of parameters used:}

$\gamma_{\text {mica }} \approx 300 \mathrm{mN} / \mathrm{m}$ is taken from $\mathrm{p} 417$ in JNI book. ${ }^{2}$

$\gamma_{T D}=27 \mathrm{mN} / \mathrm{m}$ is also from $\mathrm{JNI}$ book $^{2}$ page 418 (Table 17.1)

$\gamma_{S A M} \cong 22 \mathrm{mN} / \mathrm{m}$ is based on Langmuir measurements in our lab and is close to literature estimates $^{3}$ for a similar system.

$\gamma_{S A M / T D} \cong 0.3 \mathrm{mN} / \mathrm{m}$ results from equation 17.6 in $\mathrm{JNI}_{\text {book }^{2}}$ (page 417 ) which is written here as equation $\mathrm{S} 1$ :

$\gamma_{12}=\gamma_{1}+\gamma_{2}-2 \sqrt{\gamma_{1}^{d} \gamma_{2}^{d}}$

Putting $\gamma_{S A M} \cong 22 \mathrm{mN} / \mathrm{m}$ and $\gamma_{T D}=27 \mathrm{mN} / \mathrm{m}$ results in $\gamma_{S L} \cong 0.3 \mathrm{mN} / \mathrm{m}$.

\section{Spreading of pure TD on ODA coated mica}

The pure TD drop on the ODA coated surface attains same surface area as a retracted ODA/TD drop and remains unchanged (no spreading or retraction) as shown in fig. S2. 


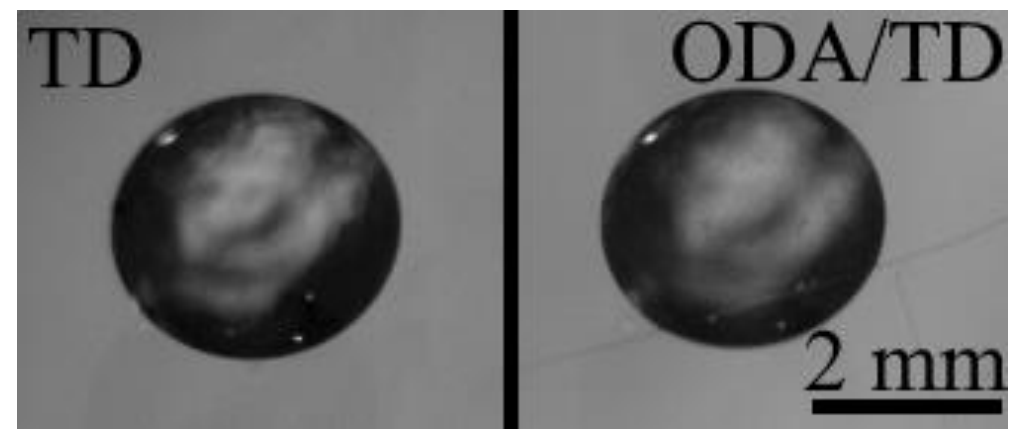

Figure S2: Snapshots of tetradecane (TD) retracted drop on ODA coated surface (left) and ODA/TD retracted drop on freshly cleaved mica surface (right).

\section{References}

(1) Harkins, W. D.; Feldman, A. FILMS. THE SPREADING OF LIQUIDS AND THE SPREADING COEFFICIENT. J. Am. Chem. Soc. 1922, 44 (12), 2665-2685.

(2) Israelachvili, J. Intermolecular and Surface Forces; Elsevier, 2011.

(3) Tadmor, R.; Rosensweig, R. E.; Frey, J.; Klein, J. Resolving the Puzzle of Ferrofluid Dispersants. Langmuir 2000, 16 (24), 9117-9120. 